Research Article

\title{
Computational Identification of Tumor Suppressor Genes Based on Gene Expression Profiles in Normal and Cancerous Gastrointestinal Tissues
}

\author{
Qingrong Sun $\mathbb{C}^{1,2}$ Md. Nazim Uddin $\mathbb{D}^{2,3}$ Mengyuan Li, ${ }^{2,3}$ Xiaosheng Wang $\mathbb{D},{ }^{2,3}$ \\ and Maode Lai ${ }^{1,2}$ \\ ${ }^{1}$ Department of Basic Medicine, School of Basic Medicine and Clinical Pharmacy, China Pharmaceutical University, \\ Nanjing 211198, China \\ ${ }^{2}$ Cancer Genomics Research Center, School of Basic Medicine and Clinical Pharmacy, China Pharmaceutical University, \\ Nanjing 211198, China \\ ${ }^{3}$ Biomedical Informatics Research Lab, School of Basic Medicine and Clinical Pharmacy, China Pharmaceutical University, \\ Nanjing 211198, China
}

Correspondence should be addressed to Xiaosheng Wang; xiaosheng.wang@cpu.edu.cn and Maode Lai; lmd@cpu.edu.cn

Received 25 March 2020; Accepted 14 May 2020; Published 22 July 2020

Guest Editor: Yue Zhang

Copyright (c) 2020 Qingrong Sun et al. This is an open access article distributed under the Creative Commons Attribution License, which permits unrestricted use, distribution, and reproduction in any medium, provided the original work is properly cited.

Cancer prevails in various gastrointestinal (GI) organs, such as esophagus, stomach, and colon. However, the small intestine has an extremely low cancer risk. It is interesting to investigate the molecular cues that could explain the significant difference in cancer incidence rates among different GI tissues. Using several large-scale normal and cancer tissue genomics datasets, we compared the gene expression profiling between small intestine and other GI tissues and between GI cancers and normal tissues. We identified 17 tumor suppressor genes (TSGs) which showed significantly higher expression levels in small intestine than in other GI tissues and significantly lower expression levels in GI cancers than in normal tissues. These TSGs were mainly involved in metabolism, immune, and cell growth signaling-associated pathways. Many TSGs had a positive expression correlation with survival prognosis in various cancers, confirming their tumor suppressive function. We demonstrated that the downregulation of many TSGs was associated with their hypermethylation in cancer. Moreover, we showed that the expression of many TSGs inversely correlated with tumor purity and positively correlated with antitumor immune response in various cancers, suggesting that these TSGs may exert their tumor suppressive function by promoting antitumor immunity. Furthermore, we identified a transcriptional regulatory network of the TSGs and their master transcriptional regulators (MTRs). Many of MTRs have been recognized as tumor suppressors, such as HNF4A, ZBTB7A, p53, and RUNX3. The TSGs could provide new molecular cues associated with tumorigenesis and tumor development and have potential clinical implications for cancer diagnosis, prognosis, and treatment.

\section{Introduction}

The gastrointestinal (GI) tract is a highly organized organ system in human with many important functions, such as the absorption of food and nutrients, endocrine secretion, and resistance to microorganism invasion. The GI cancers prevail and account for a large number of cancer deaths worldwide, of which colorectal, stomach, esophagus, liver, and pancreatic cancers are the most common [1]. However, the small intestine, an important GI tract organ for the food and nutrients absorption, has much lower cancer incidence compared to other GI tract organs. Therefore, it is interesting to investigate what molecular features may explain the significantly lower cancer incidence rate in small intestine than in other GI tract organs. With the advancement of genomics technology, a large volume of cancer and normal tissue genomics data has been produced that would enable us to investigate the association between genomic features 
and the significantly differential cancer incidence rates among different human tissues.

In this study, we compared the gene expression profiling between the tissue (small intestine) with a low cancer risk and the tissues (colon, stomach, and esophagus) with a high cancer risk using the Genotype-Tissue Expression (GTEx) data [2]. We identified the genes that were significantly differentially expressed between both groups of tissues. Moreover, we compared the gene expression profiling between cancer and normal tissues in colon, stomach, and esophagus cancer cohorts using The Cancer Genome Atlas (TCGA) data and identified differentially expressed genes (DEGs) between cancer and normal tissues. We obtained common DEGs between the results from both analyses and divided them into tumor promoter genes (TPGs) and tumor suppressor genes (TSGs). The TPGs were those that are more highly expressed in colon, stomach, and esophagus than in small intestine and more highly expressed in colon, stomach, and esophagus cancers than in their normal tissues. By contrast, the TSGs were those that are more lowly expressed in colon, stomach, and esophagus than in small intestine and more lowly expressed in colon, stomach, and esophagus cancers than in their normal tissues. Figure 1 is a summary of the analysis pipeline for identifying TPGs and TSGs. Furthermore, we verified these results using several Gene Expression Omnibus (GEO) datasets [3]. The downstream analysis of the genes identified was performed based on TCGA data.

\section{Materials and Methods}

2.1. Materials. The gene expression profiling of normal tissues (small intestine, colon, stomach, and esophagus) was downloaded from GTEx (https://gtexportal.org/home/) and GEO (https://www.ncbi.nlm.nih.gov/gds) databases. The gene expression profiling of colon, stomach, and esophagus cancers and their normal tissues were downloaded from TCGA (https://portal.gdc.cancer.gov/). In addition, we obtained the gene expression profiling and clinical immunotherapy response data of two melanoma cohorts (Nathanson et al. [4] and Roh et al. [5]) from the associated publications. The sample size of cancer and normal tissues are presented in Supplementary Table S1.

2.2. Identification of Differentially Expressed Genes. We identified differentially expressed genes (DEGs) between two classes of samples using Student's $t$-test. The false discovery rate (FDR) estimated by the Benjamini and Hochberg (BH) method [6] was used to adjust for multiple tests. The threshold of FDR $<0.05$ and mean gene-expression foldchange $>1.5$ was used to identify the DEGs between two classes of samples.

2.3. Identification of TPGs and TSGs. Based on the GTEx datasets, we identified three sets of more highly expressed genes and three sets of more lowly expressed genes in small intestine by comparing small intestine tissue versus colon tissue, intestine tissue versus gastric tissue, and intestine tissue versus esophagus tissue. We obtained the set of genes common in the three sets of more highly expressed genes in small intestine (termed as SI-HEGs) and the set of genes common in the three sets of more lowly expressed genes in small intestine (termed as SILEGs). Furthermore, based on the TCGA datasets, we identified three sets of upregulated genes and three sets of downregulated genes in cancer by comparing colon cancer versus colon tissue, gastric cancer versus gastric tissue, and esophagus cancer versus esophagus tissue. We termed the set of genes consistently upregulated in the three cancer types as Ca-HEGs and the set of genes consistently downregulated in the three cancer types as Ca-LEGs. We defined TPGs as the set of genes overlapping between SI-LEGs and Ca-HEGs and TSGs as the set of genes overlapping between SI-HEGs and Ca-LEGs.

2.4. Survival Analyses. We compared overall survival (OS) and disease-free survival (DFS) between cancer patients with higher gene expression levels (>median) and cancer patients with lower gene expression levels $(<$ median) and between cancer patients with higher tumor immunity ( $>$ median) and cancer patients with lower tumor immunity ( $<$ median). The tumor immunity was calculated by the ABSOLUTE algorithm [7]. The significance of survival time differences was evaluated by the log-rank test using a threshold of $P<0.05$. Kaplan-Meier curves were used to show the survival time differences. The survival analyses were performed in the TCGA datasets.

2.5. Correlation of Gene Expression Levels with Tumor Purity, Tumor Immune Cell Infiltration Levels, and Immunotherapy Response. We evaluated the correlations of gene expression levels [8] with tumor purity and the abundance of tumor immune cell (CD8+ T cells and dendritic cells) infiltration using TIMER [9]. In both melanoma cohorts (Nathanson et al. [4] and Roh et al. [5]), we divided cancer samples into two groups based on the median expression levels of TSGs and compared the immunotherapy response rates between them.

2.6. Comparison of the Methylation Levels of TSGs between Tumor and Normal Tissues. We compared the mean gene promoter methylation levels ( $\beta$ values) between tumor and normal tissues in 18 TCGA cancer types and used the linear regression model to evaluate the correlation between gene expression levels and mean gene promoter methylation levels in these cancer types using MethHC [10].

2.7. Identification of Master Transcriptional Regulators (MTRs) of TSGs. We used iRegulon [11], a Java-based plugin in Cytoscape, to identify the MTRs that regulate the TSGs. iRegulon uses a large collection of transcription factor (TF) motifs and ChIP-seq tracks to identify the target genes of TFs on the basis of the normalized enrichment score (NES). 


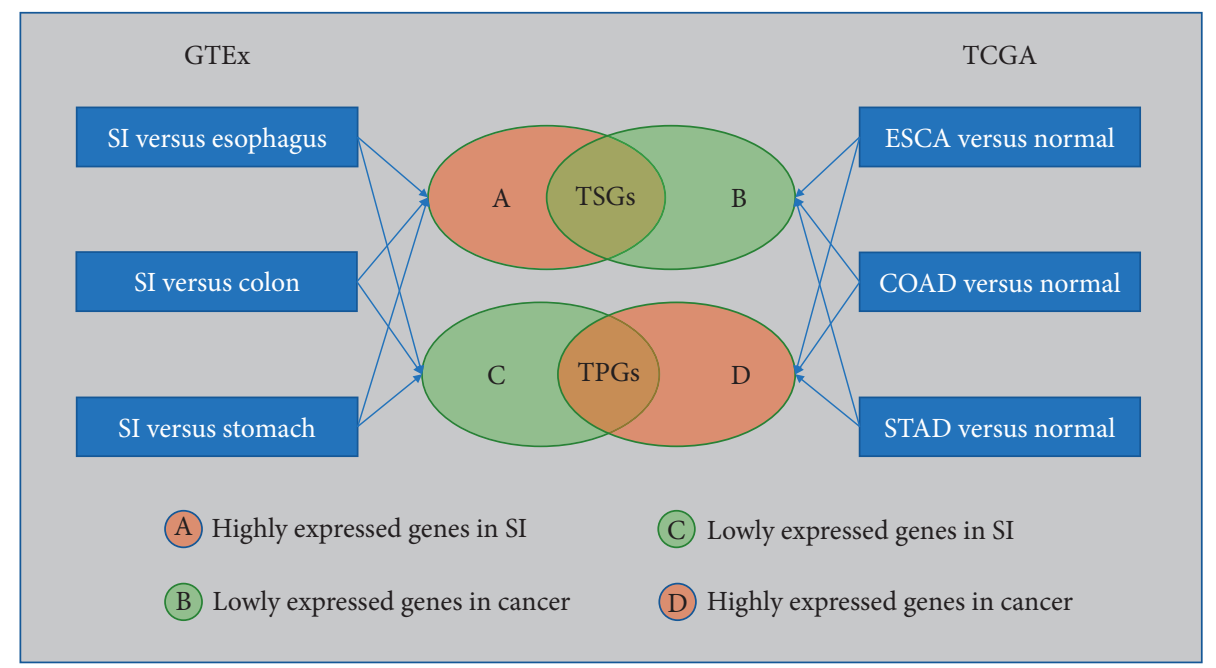

Figure 1: The analysis pipeline for identifying tumor promoter genes (TPGs) and tumor suppressor genes (TSGs). The differentially expressed genes (DEGs) between two classes of samples were identified using the threshold of Student's $t$-test adjusted $P$ value (FDR) $<0.05$ and mean gene-expression fold-change $>1.5$. The FDR was calculated by the Benjamini and Hochberg (BH) method [6]. SI: small intestine; FDR: false discovery rate.

\section{Results}

3.1. Identification of TSGs. We identified a total of 17 TSGs and zero TPGs. The 17 TSGs included FAM177B, TMEM25, ANGPTL6, TREH, RASGRP2, SLC28A2, MADCAM1, XPNPEP2, SEPP1, CCL21, PNOC, GUCA2B, GSTA2, G6PC, FCER2, CBFA2T3, and BMP5. Among the 17 TSGs, 8 genes were found to be associated with certain KEGG [12] pathways (Table 1). These pathways are mainly involved in metabolism (starch and sucrose, cytochrome P450, glutathione, galactose, and glycolysis), immune (chemokine signaling, cytokine-cytokine receptor interaction, intestinal immune network for IgA production, adipocytokine, insulin signaling, and hematopoietic cell lineage), and cell proliferation, differentiation, and migration (MAPK signaling, cell adhesion molecules, and TGF- $\beta$ signaling). These results indicate that these gene signatures are importantly involved in tumor suppression by the regulation of metabolism, immune, and cell growth signaling-associated pathways.

Moreover, we found that a majority of the 17 TSGs showed significantly lower expression levels in various cancers apart from colon, stomach, and esophagus cancers than in normal tissues. For example, RASGRP2 was downregulated in 20 TCGA cancer types (Figure 2). CCL21 had lower expression levels in 18 TCGA cancer types (Supplementary Figure S1). CBFA2T3 and XPNPEP2 were downregulated in 15 and 10 TCGA cancer types (Supplementary Figures S2 and S3). These results suggest that the TSGs may play crucial roles in tumor suppression in a wide variety of cancer types.

Furthermore, we found that the elevated expression of many TSGs correlated with a better survival prognosis in various cancers. For example, the upregulation of RASGRP2 was consistently associated with a better overall survival (OS) or disease-free survival (DFS) in 12 cancer types, including breast invasive carcinoma (BRCA), cervical and endocervical cancers (CESC), cholangiocarcinoma (CHOL), colon adenocarcinoma (COAD), head and neck squamous cell carcinoma (HNSC), liver hepatocellular carcinoma (LIHC), lung adenocarcinoma (LUAD), pancreatic adenocarcinoma (PAAD), sarcoma (SARC), skin cutaneous melanoma (SKCM), thymoma (THYM), and uterine corpus endometrial (UCEC) (Figure 3). The elevated expression of CBFA2T3 was consistently associated with a better OS or DFS in 10 cancer types, including CHOL, COAD, HNSC, kidney chromophobe $(\mathrm{KICH})$, brain lower-grade glioma (LGG), LIHC, LUAD, SARC, thyroid carcinoma (THCA), and THYM (Supplementary Figure S4). Again, these results suggest the tumor suppressive function of TSGs.

The depressed expression of TSGs is associated with the hypermethylation of these genes in cancer.

We compared the DNA methylation levels of TSGs between cancer and normal tissues and found that many TSGs exhibited significantly higher methylation levels in various cancers. For example, the methylation levels of RASGRP2 promoter were significantly higher in 18 TCGA cancer types than in their normal tissues (Figure S5). The promoter region of CCL21 had significantly higher methylation levels in 17 TCGA cancer types (Supplementary Figure S7). Furthermore, linear regression analysis showed that the methylation levels of TSGs had a significant inverse correlation with the expression levels of TSGs in many cancer types. For example, RASGRP2 methylation levels significantly inversely correlated with its expression levels in stomach adenocarcinoma (STAD), PAAD, and prostate adenocarcinoma (PRAD) with the absolute correlation coefficient not less than 0.3 (Supplementary Figures S6 and S7). In addition, previous studies have shown that several TSGs had significantly higher methylation levels in cancer, such as CBFA2T3 [13] and TMEM25 [14]. These results suggest that the downregulation of many TSGs is associated with their elevated methylation levels in cancer. 
TABle 1: The 17 tumor suppressor genes (TSGs) and their associated pathways.

\begin{tabular}{|c|c|c|c|}
\hline Entrez ID & Symbol & Name & Pathway \\
\hline 11181 & TREH & Trehalase (brush-border membrane glycoprotein) & Starch and sucrose metabolism \\
\hline 10235 & RASGRP2 & RAS guanyl releasing protein 2 (calcium and DAG-regulated) & $\begin{array}{l}\text { Chemokine signaling pathway } \\
\text { MAPK signaling pathway }\end{array}$ \\
\hline 8174 & $M A D C A M 1$ & Mucosal vascular addressin cell adhesion molecule 1 & $\begin{array}{l}\text { Cell adhesion molecules (CAMs) } \\
\text { Intestinal immune network for IgA production }\end{array}$ \\
\hline 6366 & CCL21 & Chemokine (C-C motif) ligand 21 & $\begin{array}{l}\text { Chemokine signaling pathway } \\
\text { Cytokine-cytokine receptor interaction } \\
\text { Drug metabolism-cytochrome P450 }\end{array}$ \\
\hline 2939 & GSTA2 & Glutathione S-transferase alpha 2 & $\begin{array}{l}\text { Glutathione metabolism } \\
\text { Metabolism of xenobiotics by cytochrome P450 } \\
\text { Adipocytokine signaling pathway } \\
\text { Galactose metabolism }\end{array}$ \\
\hline 2538 & G6PC & Glucose-6-phosphatase, catalytic subunit & $\begin{array}{l}\text { Glycolysis/gluconeogenesis } \\
\text { Insulin signaling pathway } \\
\text { Starch and sucrose metabolism }\end{array}$ \\
\hline 2208 & FCER2 & Fc fragment of IgE, low affinity II, receptor for CD23 & Hematopoietic cell lineage \\
\hline 653 & BMP5 & Bone morphogenetic protein 5 & TGF-beta signaling pathway \\
\hline
\end{tabular}

The higher expression levels of TSGs are associated with lower tumor purity and more active antitumor immune response in cancer.

We found that the expression levels of TSGs tended to inversely correlate with tumor purity in various cancers. For example, the RASGRP2 expression levels were inversely associated with tumor purity in 25 TCGA cancer types/subtypes with the Spearman rank correlation coefficient (cor) not greater than -0.3 (Figure 4 ). The expression levels of CCL21 had a significant inverse correlation with tumor purity in 17 TCGA cancer types (cor $\leq-0.3$ ) (Supplementary Figure S8). These results indicate that the higher expression levels of TSGs may correlate with more nontumor components in cancer. In fact, the expression levels of TSGs were likely to have a positive correlation with antitumor immune signatures in various cancers. For example, the RASGRP2 expression levels positively correlated with the enrichment levels of CD8+ T cells in 14 TCGA cancer types/subtypes and with the enrichment levels of dendritic cells in 26 TCGA cancer types/subtypes (cor $\geq 0.3$ ) (Figure 5). The expression levels of CCL21 had a significant positive correlation with the enrichment levels of CD8+ T cells and dendritic cells in 5 and 6 TCGA cancer types, respectively (cor $\geq 0.3$ ) (Supplementary Figure S9). Previous studies also showed that the expression of certain TSGs could promote antitumor immunity in diverse cancers, such as CCL21 [15-17], MADCAM1 [18], and FCER2 [19]. Again, these results suggest that the elevated expression of TSGs is associated with a favorable prognosis in cancer since the higher levels of tumor-infiltrating lymphocytes (TILs) are associated with improved survival in cancer patients $[20,21]$.

Since the elevated expression of TSGs was associated with the higher levels of TILs in tumor and the TILs levels were a positive predictor for cancer immunotherapy response (ITR) [22], the expression levels of TSGs could be positively associated with ITR in cancer. To prove this hypothesis, we examined the correlation between the expression levels of TSGs and ITR in two cancer (melanoma) cohorts (Nathanson et al.'s cohort [4] and Roh et al.'s cohort [5]) with anti-CTLA-4/PD-1 immunotherapy. We found that the higher GSTA2 expression levels were associated with a significantly higher ITR in Nathanson et al.'s cohort (Fisher's exact test, $P=0.036, \mathrm{OR}=8.91$ ) and that the expression of CCL21 and MADCAM1 positively correlated with ITR in Roh et al.'s cohort (Fisher's exact test, $P=0.002$, $\mathrm{OR}=8.07$ for CCL21 and $P=0.058$, $\mathrm{OR}=4.55$ for $M A D$ CAM1) (Table 2). These results proved the hypothesis that the expression of TSGs is capable of promoting ITR in cancer.

3.2. Master Transcriptional Regulators (MTRs) of TSGs. To understand the regulatory mechanism underlying the different cancer risk in varying tissues, we identified 34 MTRs that significantly regulated the 17 TSGs (NES >3) (Figure 6). Among the 34 MTRs, HNF4A (hepatocyte nuclear factor 4 alpha) was the most highly enriched which regulated 14 TSGs. HNF4A plays a role in the development of multiple organs including intestines, liver, and kidney and functions as a repressor of cell proliferation [23]. The reduced expression of HNF4A has been associated with tumorigenesis in various cancers $[24,25]$. This is consistent with the tumor suppressive function of the TSGs regulated by HNF4A. The second highly enriched MTR of TSGs was ZBTB7A regulating 11 TSGs. ZBTB7A acts as a tumor suppressor by repressing the expression of key genes in tumor glycolysis [26] and negatively regulating TGF- $\beta$ pathway [27]. Previous studies have revealed its tumor suppressor role in a wide variety of cancers [28-30]. Again, this is in line with the tumor suppressive function of the TSGs regulated by ZBTB7A. Some other MTRs have been also identified as tumor suppressors, such as p53 [31-33] and RUNX3 [34-36]. Collectively, these results suggest that the identification of MTRs of TSGs may provide insights into the regulatory mechanism underlying the different cancer risks in different tissues and cancer development. 


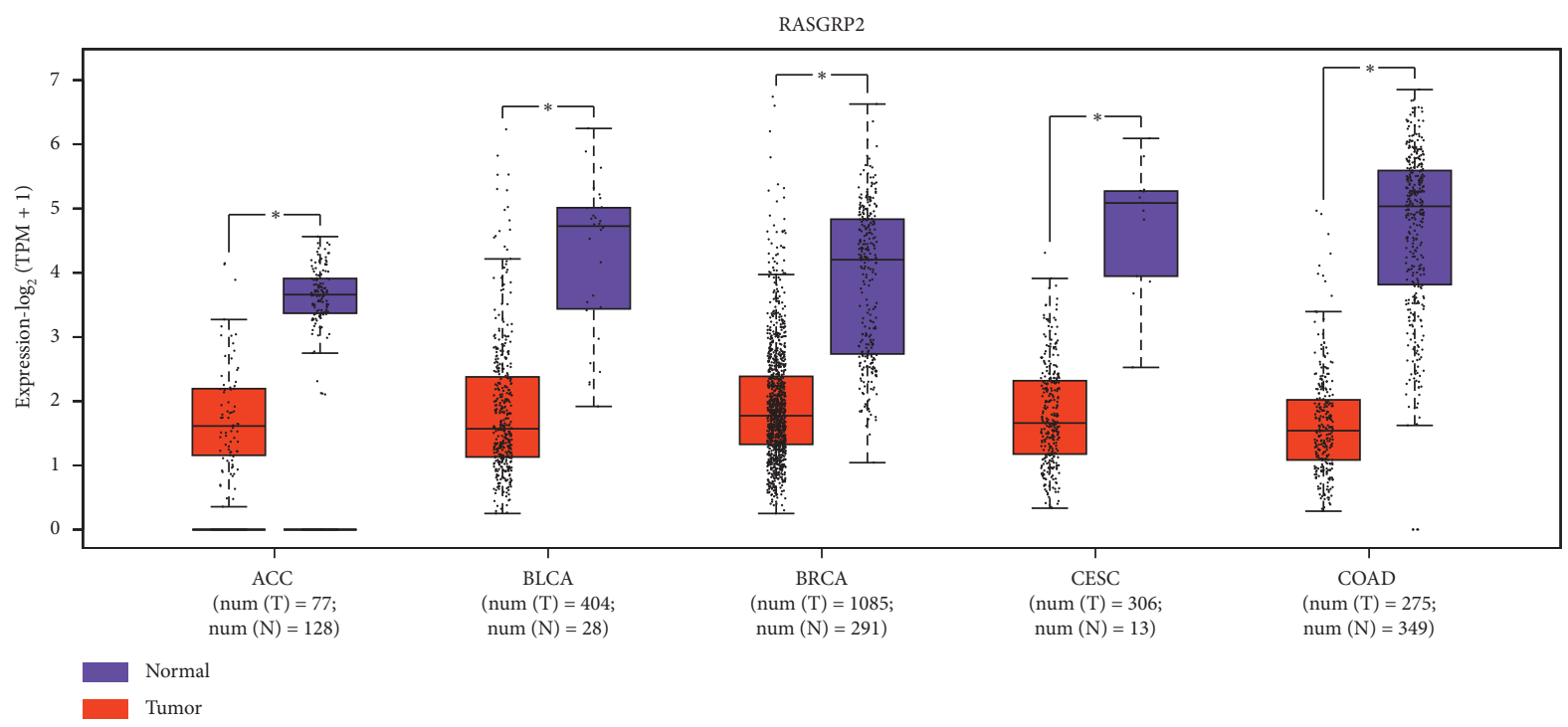

(a)

RASGRP2

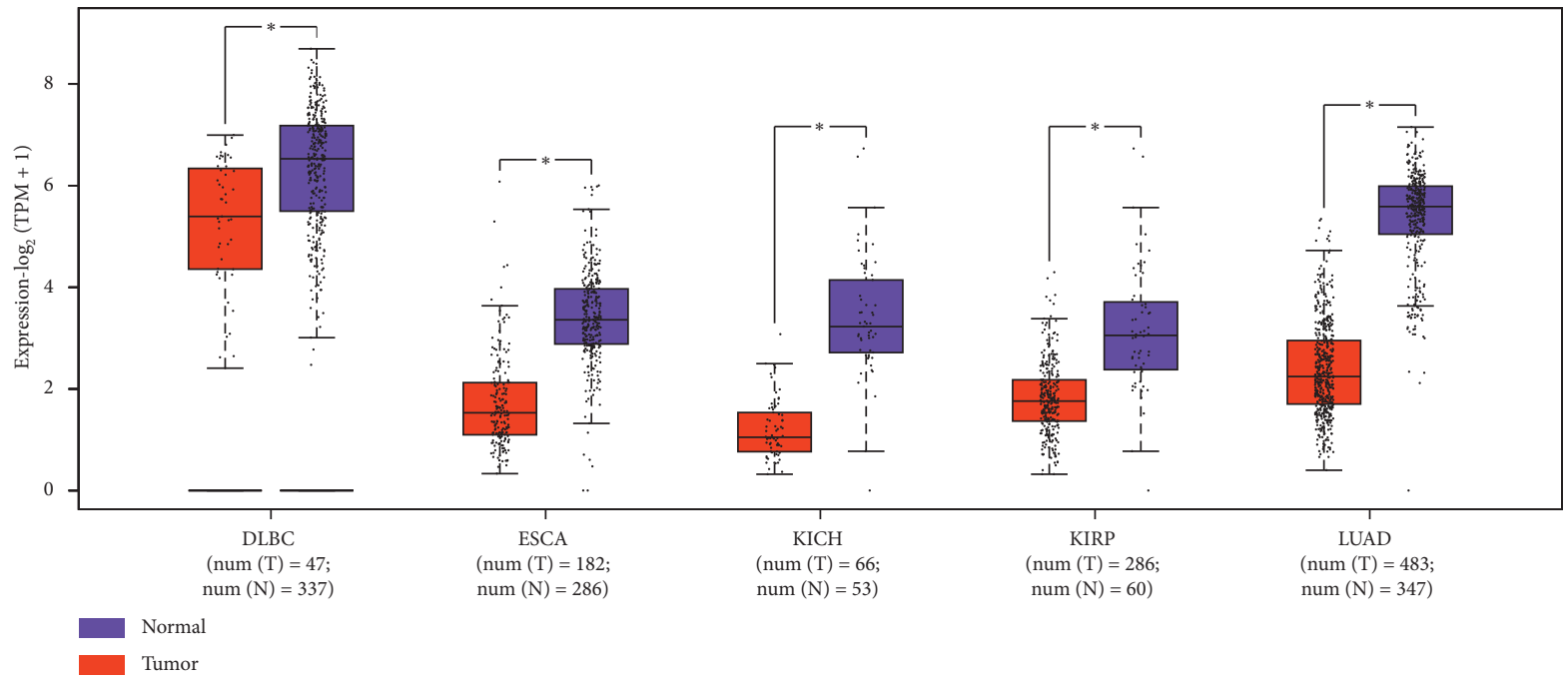

(b)

RASGRP2

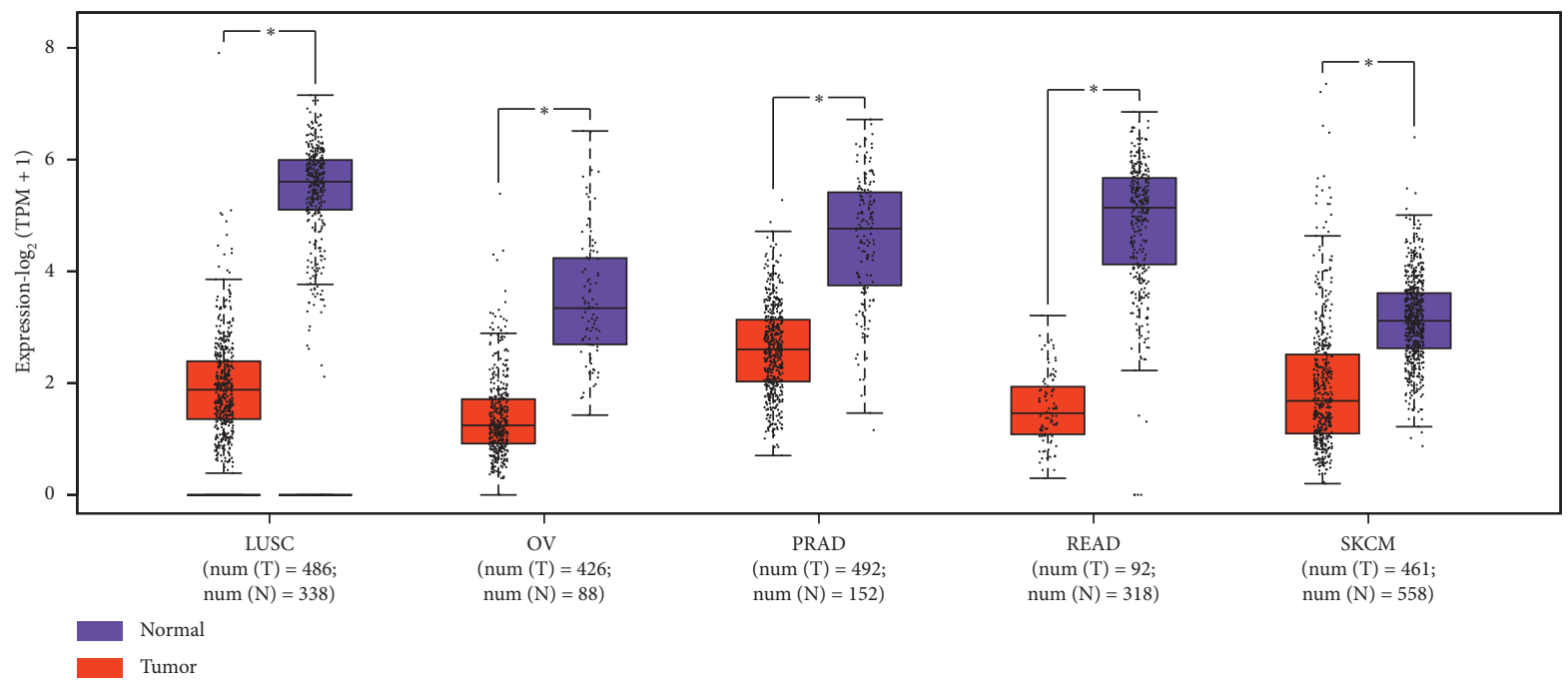

(c)

FIgURE 2: Continued. 
RASGRP2

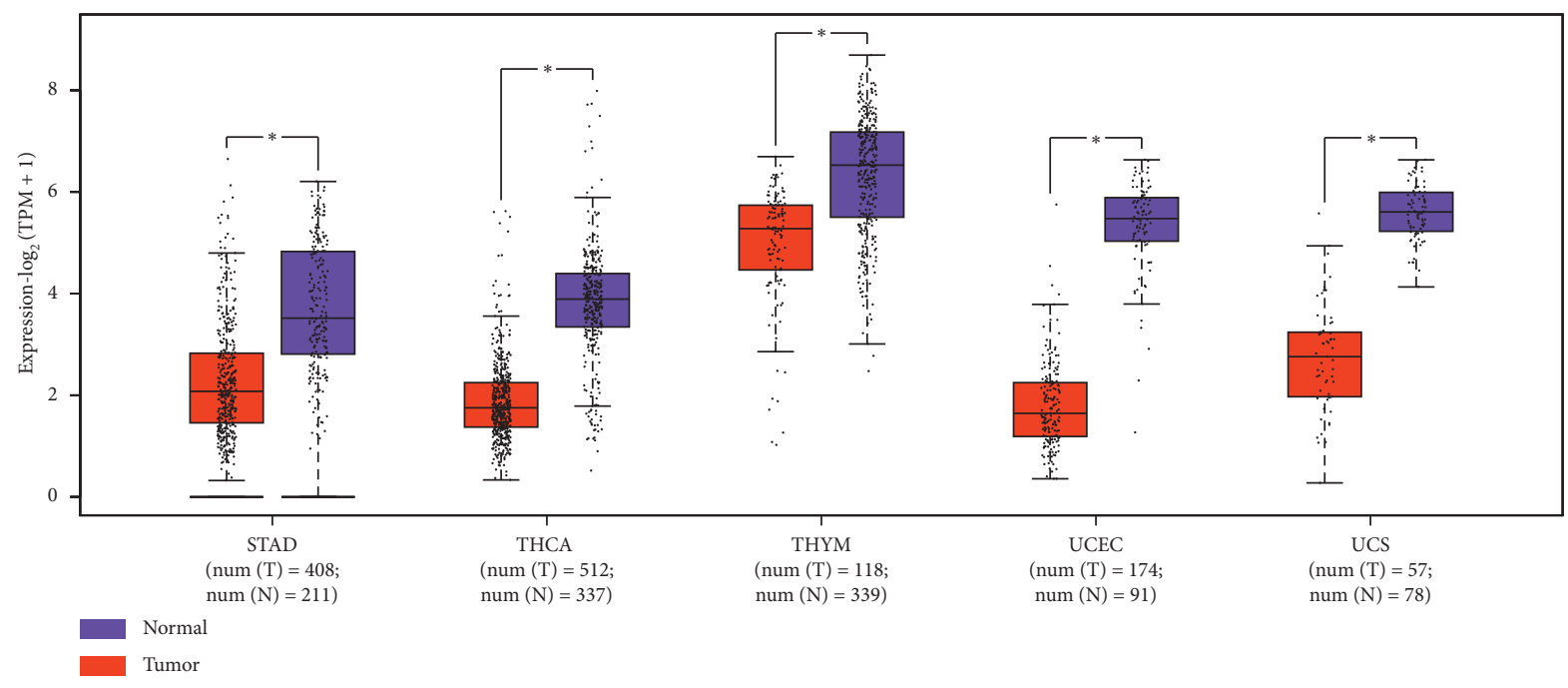

(d)

FIGURE 2: RASGRP2 is downregulated in cancer. RASGRP2 has significantly lower expression levels in various cancers than in normal tissues (Student's $t$-test, $P<0.001$ ). TPM: transcripts per kilobase million. ${ }^{* * *} P<0.001$; it also applies to the following figures.
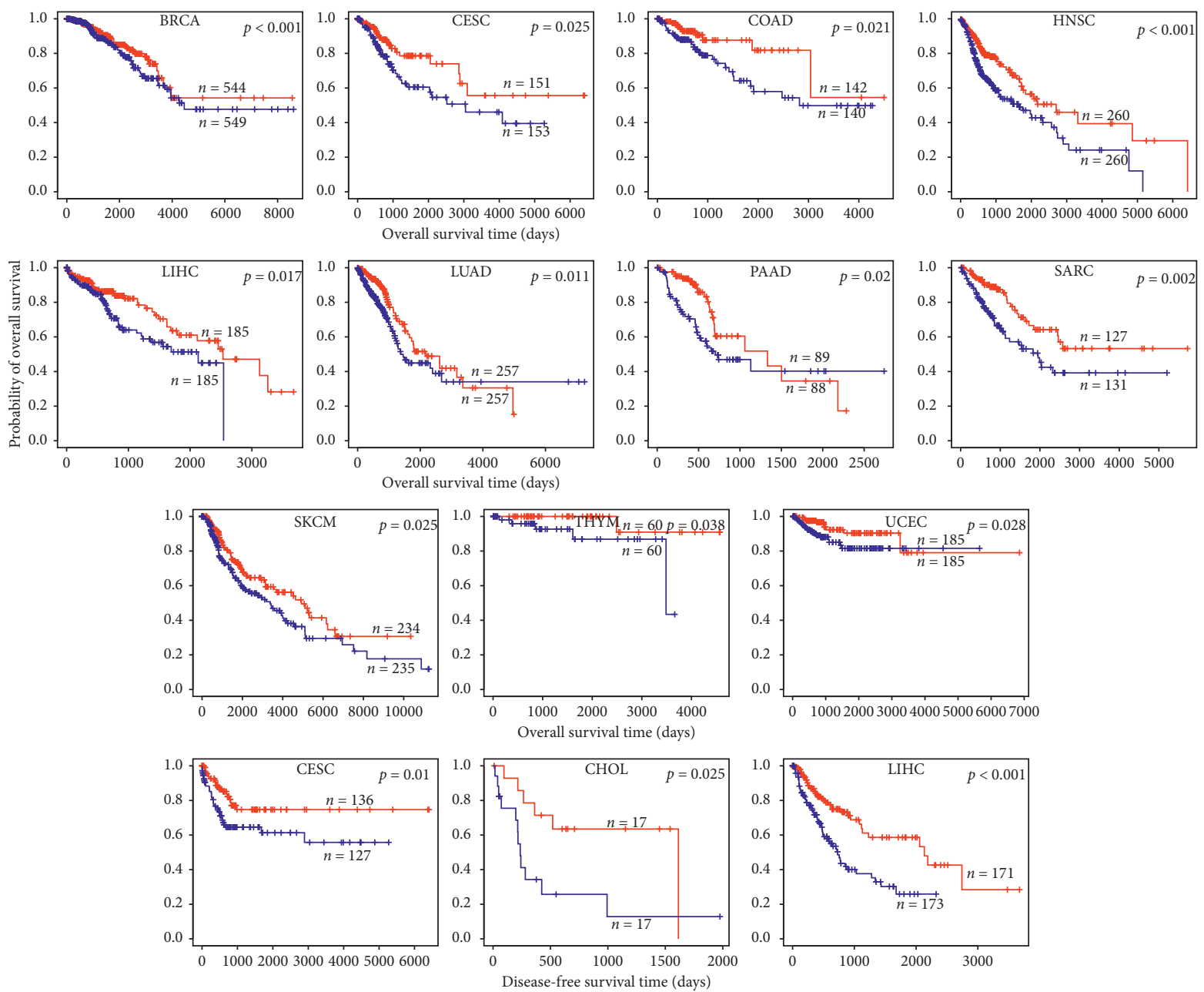

$\square$ RASGRP2 higher expression

FIGURE 3: Downregulation of RASGRP2 is associated with a worse survival prognosis in various cancers. Kaplan-Meier survival curves show that the lower expression of $R A S G R P 2$ is associated with a worse overall or disease-free survival in various cancers $(l o g$-rank test, $P<0.05)$. 

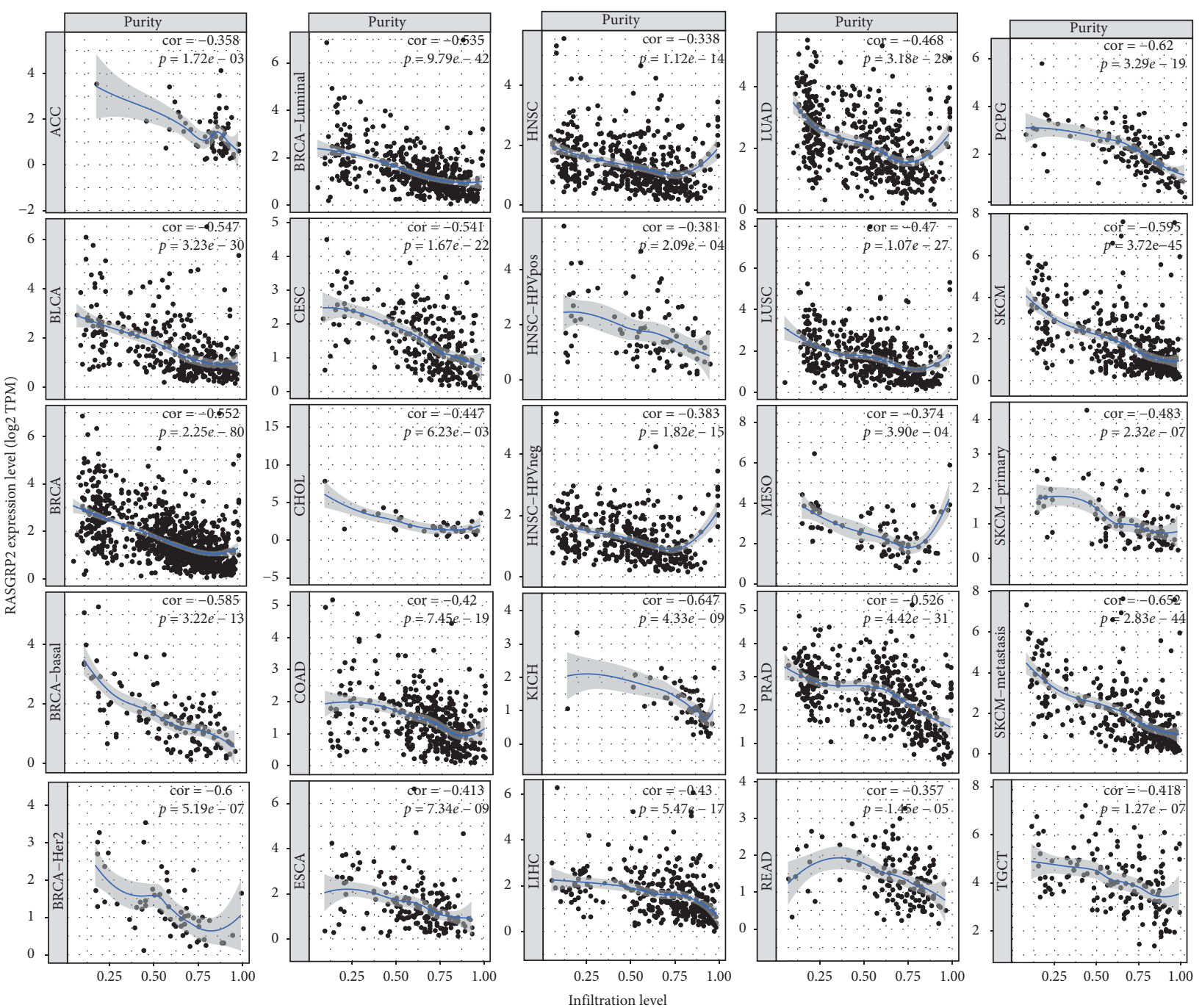

FIGURE 4: Correlations of the expression levels of tumor suppressor genes (TSGs) with tumor purity in cancer. The RASGRP2 expression levels were inversely associated with tumor purity in 20 TCGA cancer types/subtypes.

\section{Discussion}

Cancer prevails in variously different human organs, such as lung, colon, stomach, esophagus, liver, pancreas, brain, head and neck, breast, and kidney. However, some human organs have an extremely low cancer risk, such as small intestine, spleen, and heart. Although a recent study has proposed that the total number of stem cell divisions largely explained the cancer risk in different tissues [37], it cannot explain why some tissues with a large number of stem cell divisions have a low cancer risk, such as small intestine. Thus, it is necessary to investigate the other molecular cues that could explain the different cancer risk among different tissues. We identified 17 TSGs which showed significantly higher expression levels in small intestine than in other GI tissues including esophagus, stomach, and colon. Moreover, these genes were more lowly expressed in GI cancers than in GI normal tissues and were also downregulated in many other cancer types relative to their normal control, suggesting their tumor suppressor role. The tumor suppressive function of these
TSGs was further confirmed by the fact that the elevated expression of many TSGs was associated with a better survival prognosis in various cancers. Furthermore, we revealed that the downregulation of many TSGs was associated with their promoter hypermethylation in cancer, demonstrating the important relationship between DNA methylation and cancer [38]. Pathway analysis showed that these TSGs were mainly involved in metabolism, immune, and cell growth signaling-associated pathways. Interestingly, the expression of many TSGs inversely correlated with tumor purity and positively correlated with antitumor immune cell infiltration levels in a wide variety of cancers, suggesting that these TSGs may exert their tumor suppressive function by promoting antitumor immunity. Moreover, the higher expression levels of certain TSGs, including GSTA2, CCL21, and MADCAM1, were associated with a significantly higher ITR in cancer. This could be attributed to the higher levels of TILs in the tumors highly expressing these TSGs. In addition, we identified 34 MTRs of TSGs, many of which have been recognized as tumor 

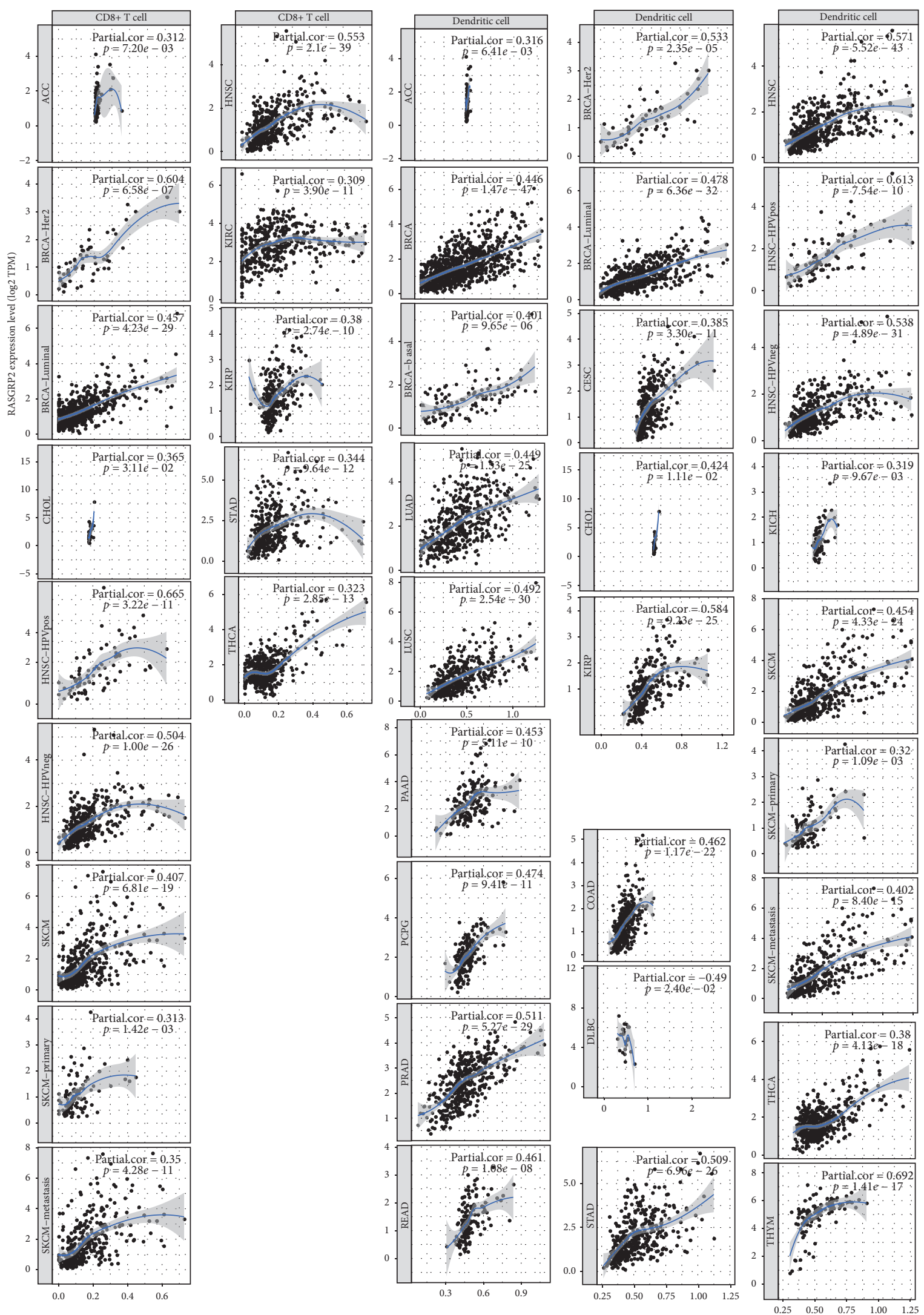

FIGURE 5: Correlations of the expression levels of tumor suppressor genes (TSGs) with immune cell infiltration levels in cancer. The RASGRP2 expression levels were positively associated with the enrichment levels of immune cells (CD8+ T cells and dendritic cells) in multiple TCGA cancer types/subtypes. Spearman's rank correlation test $P$ values and correlation coefficients are shown. RSEM : RNA-Seq by Expectation Maximization [8]. 
TABLE 2: Correlations of the expression levels of tumor suppressor genes (TSGs) with immunotherapy response in cancer. Anti-CTLA-4/ PD-1 immunotherapy response rates in melanoma patients with high or low expression of three TSGs. Fisher's exact test $P$ values are shown.

\begin{tabular}{|c|c|c|c|c|c|c|}
\hline Cohorts & \multicolumn{2}{|c|}{$\begin{array}{c}\text { Nathanson et al.'s } \\
\text { cohort }\end{array}$} & \multicolumn{2}{|c|}{ Roh et al.'s cohort } & \multicolumn{2}{|c|}{ Roh et al.'s cohort } \\
\hline Gene symbol & \multicolumn{2}{|c|}{ GSTA2 } & \multicolumn{2}{|c|}{ MADCAM1 } & \multicolumn{2}{|c|}{ CCL21 } \\
\hline Expression & Low & High & Low & High & Low & High \\
\hline Response (\%) & 16.67 & 66.67 & 29.73 & 66.67 & 16.00 & 61.90 \\
\hline No response $(\%)$ & 83.33 & 33.33 & 70.27 & 33.33 & 84.00 & 38.10 \\
\hline Fisher's exact test $P$ values & \multicolumn{2}{|c|}{0.036} & \multicolumn{2}{|c|}{0.058} & \multicolumn{2}{|c|}{0.002} \\
\hline
\end{tabular}

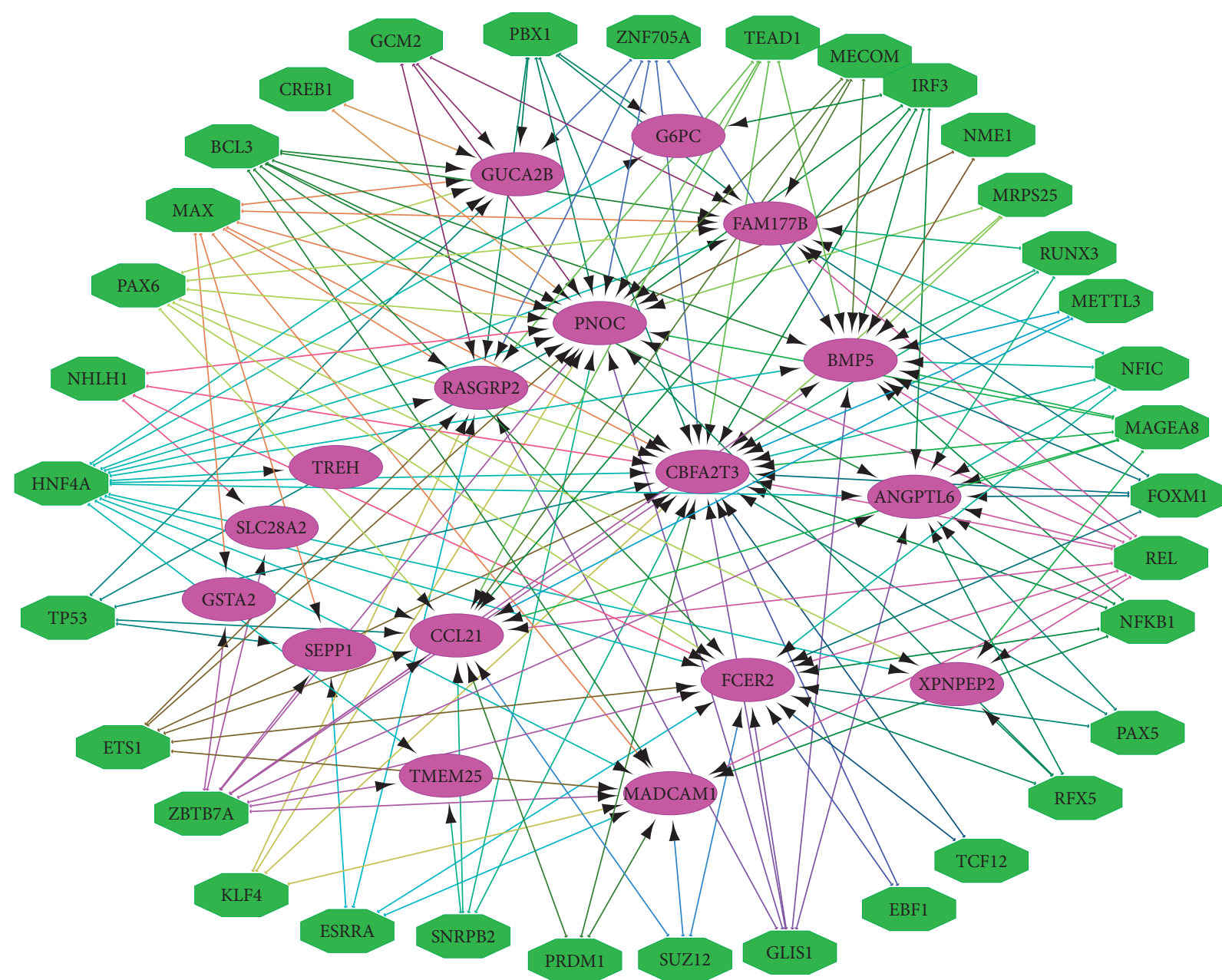

FIGURE 6: Regulatory network of 17 tumor suppressor genes (TSGs) and their master transcriptional regulators (MTRs). The purple nodes indicate TSGs and the green nodes indicate MTRs.

suppressors, such as HNF4A, ZBTB7A, p53, and RUNX3. It suggests that the transcriptional regulatory network could also be an important approach whereby the TSGs inhibit tumor development.

Our results showed that the elevated expression of TSGs was likely associated with better survival prognosis in cancer. A possible explanation is that the expression of TSGs is positively associated with the infiltration of TILs which may improve the immune response against cancer cells. Our results also showed that the elevated expression of TSGs was likely associated with lower tumor purity whose association with prognosis is controversial. We analyzed the correlation between tumor purity and survival prognosis in 33 TCGA cancer types. We found that tumor purity had a significant positive correlation with OS in COAD, KIRP, and PRAD and with DFS in kidney renal clear cell carcinoma (KIRC), UCEC, and uveal melanoma (UVM) (log-rank test, $P<0.05)$, but have a significant negative correlation with DFS in HNSC $(P=0.035)$ (Supplementary Figure S10). These results indicate that the correlation between tumor purity and survival prognosis was not significant in most cancer types although it was positive or negative in a few cancer types.

Based on another gene expression profiling dataset from GEO [3], we found that three TSGs (G6PC, XPNPEP2, and 


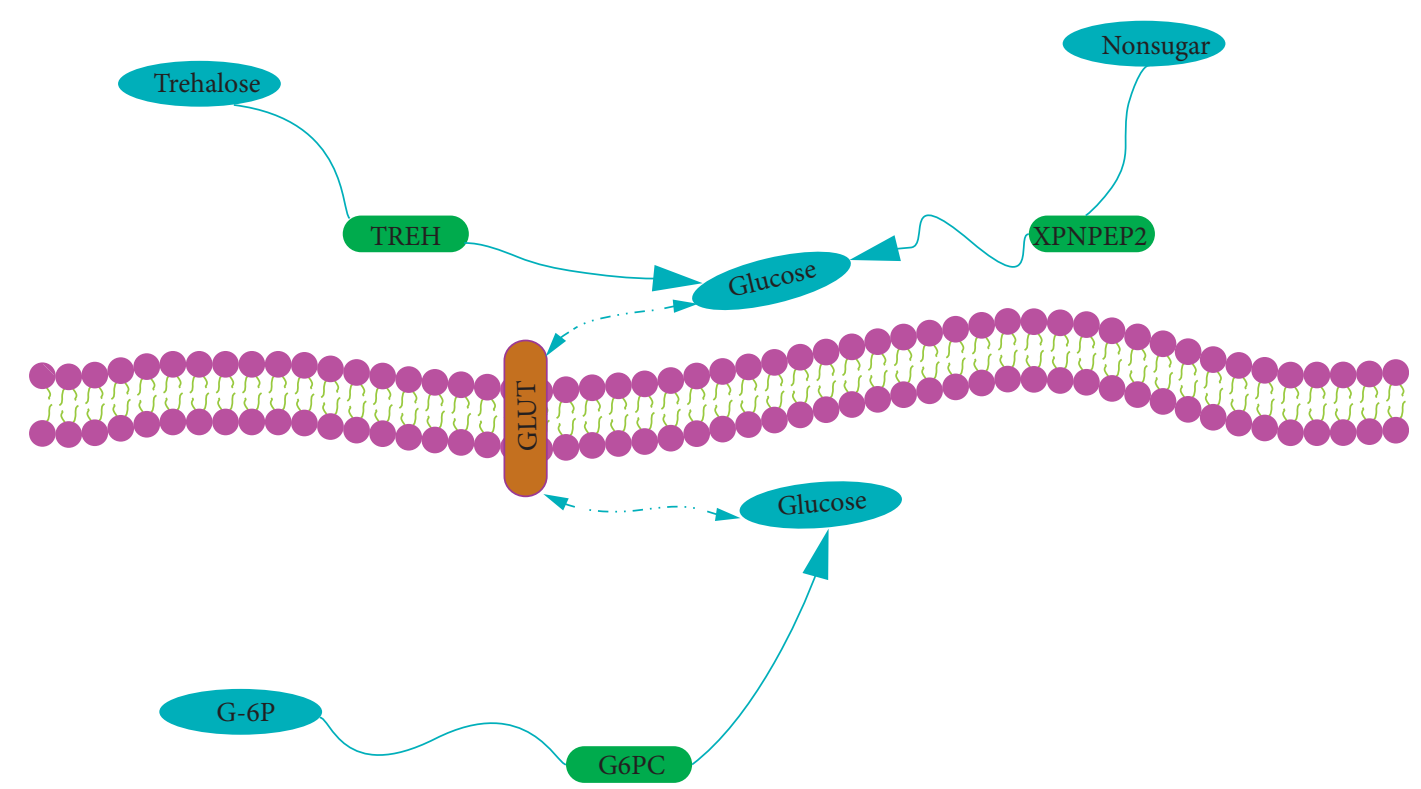

Figure 7: Three tumor suppressors (G6PC, XPNPEP2, and TREH) are involved in glucose metabolism in cells.

TREH) had significantly higher expression levels in small intestine tissue than in colon and stomach tissues, confirming the results obtained from the analysis of GTEx dataset. Using COMPARTMENTS [39], we found that G6PC was located in the endoplasmic reticulum and both XPNPEP2 and TREH were located in the plasma membrane. On the basis of the connections between pathways and proteins' subcellular location, we found that both G6PC and TREH were associated with glucose metabolism and contributed to the elevation of glucose levels. In addition, XPNPEP2 acts as a proline-specific aminopeptidase to regulate the proline concentration which is capable of affecting the glucose concentration [40]. Thus, all the three proteins can enhance the glucose concentration both inside and outside cells to inhibit glucose metabolism in cells (Figure 7). Again, these results suggest that the metabolic regulation may play a crucial role in controlling tumorigenesis and tumor development.

A literature review showed that many of the 17 TSGs have been associated with tumor suppression. For example, BMP5 (bone morphogenetic protein 5) was downregulated in breast tumors relative to normal tissues and its downregulation was associated with cancer recurrence [41]. This gene plays a role in tumor suppression via repressing TGF$\beta 1$-induced epithelial-to-mesenchymal transition [41]. CCL21 (C-C motif chemokine ligand 21) is a cytokine gene involved in immunoregulation and inflammation. CCL21 is able to exert antitumor immunity by activating both innate and adaptive immune responses $[42,43]$. The other TSGs, such as SEPP1 [44], TMEM25 [45], XPNPEP2 [46], and $G 6 P C$ [47], have been reported to play a role in tumor suppression. These prior studies lend support to our results that these TSGs are likely to be tumor suppressor genes, although further experimental verification is needed.

In conclusion, this study provides new molecular cues associated with tumorigenesis and tumor development. The identified TSGs have potential clinical implications for cancer diagnosis, prognosis, and treatment.

\section{Abbreviations}

BH: $\quad$ Benjamini and Hochberg

BRCA: Breast invasive carcinoma

CHOL: Cholangiocarcinoma

COAD: Colon adenocarcinoma

DEGs: Differentially expressed genes

DFS: Disease-free survival

DLBC: Lymphoid neoplasm diffuse large B-cell lymphoma

ESCA: Esophageal carcinoma

FDR: False discovery rate

GEO: Gene Expression Omnibus

GI: $\quad$ Gastrointestinal

GTEx: The Genotype-Tissue Expression

HNSC: Head and neck squamous cell carcinoma

ITR: Immunotherapy response

KIRC: Kidney renal clear cell carcinoma

KIRP: Kidney renal papillary cell carcinoma

LGG: Brain lower-grade glioma

LIHC: Liver hepatocellular carcinoma

LUAD: Lung adenocarcinoma

MTRs: Metabolic transcriptional regulators

NES: $\quad$ Normalized enrichment score

OS: Overall survival

PAAD: Pancreatic adenocarcinoma

SARC: Sarcoma

SI- $\quad$ Highly expressed genes in small intestine

HEGs:

SI-LEGs: Lowly expressed genes in small intestine

SKCM: Skin cutaneous melanoma

STAD: Stomach adenocarcinoma

TCGA: The Cancer Genome Atlas 
TF: Transcription factor

THCA: Thyroid carcinoma

THYM: Thymoma

TILS: Tumor-infiltrating lymphocytes

TPGs: Tumor promoter genes

TSGs: Tumor suppressor genes

UCEC: Uterine corpus endometrial carcinoma.

\section{Data Availability}

The gene expression profiling of normal tissues (small intestine, colon, stomach, and esophagus) was downloaded from GTEx (https://gtexportal.org/home/) and GEO (https://www.ncbi.nlm.nih.gov/gds) databases. The gene expression profiling of colon, stomach, and esophagus cancers and their normal tissues was downloaded from TCGA (https://portal.gdc.cancer.gov/). In addition, we obtained the gene expression profiling and clinical immunotherapy response data of two melanoma cohorts (Nathanson et al.'s cohort [4] and Roh et al.'s cohort [5]) from the associated publications.

\section{Conflicts of Interest}

The authors declare that they have no conflicts of interest regarding the publication of this paper.

\section{Acknowledgments}

This project was supported by the Key Laboratory of Disease Proteomics of Zhejiang Province.

\section{Supplementary Materials}

Table S1. Sample size of cancer and normal tissues in the datasets used in this study. Figure S1. CCL21 tends to be downregulated in cancer. Figure S2. CBFA2T3 tends to be downregulated in cancer. Figure S3. XPNPEP2 tends to be downregulated in cancer. Figure S4. Downregulation of tumor suppressor genes (TSGs) is associated with a worse survival prognosis in various cancers. Figure S5. The methylation levels of RASGRP2 promoter are higher in various cancer types than in normal tissues. Figure S6. The methylation levels of RASGRP2 promoter are inversely associated with the expression levels of RASGRP2 in cancer. Figure S7. The CCL21 promoter methylation levels are significantly upregulated in 16 TCGA cancer types compared to their normal tissues. Figure S8. Correlations of the expression levels of CCL21 with tumor purity in cancers. Figure S9. Correlations of the expression levels of CCL21 with immune cell infiltration levels in cancers. Figure S10. Correlation of tumor purity with survival prognosis in cancer. (Supplementary Materials)

\section{References}

[1] R. L. Siegel, K. D. Miller, and A. Jemal, "Cancer Statistics, 2019," CA: A Cancer Journal for Clinicians, vol. 69, no. 1, pp. 7-34, 2019.
[2] G. T. Consortium, "The genotype-tissue expression (GTEx) project,” Nature Genetics, vol. 45, no. 6, pp. 580-585, 2013.

[3] R. Edgar, M. Domrachev, and A. E. Lash, "Gene Expression Omnibus: NCBI gene expression and hybridization array data repository," Nucleic Acids Research, vol. 30, no. 1, pp. 207-210, 2002.

[4] T. Nathanson, A. Ahuja, A. Rubinsteyn et al., "Somatic mutations and neoepitope homology in melanomas treated with CTLA-4 blockade," Cancer Immunology Research, vol. 5, no. 1, pp. 84-91, 2017.

[5] W. Roh, P. L. Chen, A. Reuben et al., "Integrated molecular analysis of tumor biopsies on sequential CTLA-4 and PD-1 blockade reveals markers of response and resistance," Science Translational Medicine, vol. 9, no. 379, 2017.

[6] Y. Benjamini and Y. Hochberg, "Controlling the false discovery rate: a practical and powerful approach to multiple testing," Journal of the Royal Statistical Society: Series B (Methodological), vol. 57, no. 1, pp. 289-300, 1995.

[7] C. Scott, K. Cibulskis, E. Helman et al., "Absolute quantification of somatic DNA alterations in human cancer," Nature Biotechnology, vol. 30, no. 5, pp. 413-421, 2012.

[8] B. Li and C. N. Dewey, "RSEM: accurate transcript quantification from RNA-Seq data with or without a reference genome," BMC Bioinformatics, vol. 12, no. 1, p. 323, 2011.

[9] T. Li, J. Fan, B. Wang et al., "TIMER: a web server for comprehensive analysis of tumor-infiltrating immune cells," Cancer Research, vol. 77, no. 21, pp. e108-e110, 2017.

[10] W.-Y. Huang, S.-D. Hsu, H.-Y. Huang et al., "MethHC: a database of DNA methylation and gene expression in human cancer," Nucleic Acids Research, vol. 43, no. D1, pp. D856-D861, 2015.

[11] R. Janky, A. Verfaillie, H. Imrichova et al., "iRegulon: from a gene list to a gene regulatory network using large motif and track collections," PLoS Computational Biology, vol. 10, no. 7, Article ID e1003731, 2014.

[12] M. Kanehisa, M. Furumichi, M. Tanabe, Y. Sato, and K. Morishima, "KEGG: new perspectives on genomes, pathways, diseases and drugs," Nucleic Acids Research, vol. 45, no. D1, pp. D353-D361, 2017.

[13] A. J. Bais, A. E. Gardner, O. L. McKenzie, D. F. Callen, G. R. Sutherland, and G. Kremmidiotis, "Aberrant CBFA2T3B gene promoter methylation in breast tumors," Molecular Cancer, vol. 3, no. 1, p. 22, 2004.

[14] S. Hrasovec, N. Hauptman, D. Glavac, F. Jelenc, and M. Ravnik-Glavac, "TMEM25 is a candidate biomarker methylated and down-regulated in colorectal cancer," Disease Markers, vol. 34, no. 2, pp. 93-104, 2013.

[15] S.-C. Yang, R. K. Batra, S. Hillinger et al., "Intrapulmonary administration of CCL21 gene-modified dendritic cells reduces tumor burden in spontaneous murine bronchoalveolar cell carcinoma," Cancer Research, vol. 66, no. 6, pp. 32053213, 2006.

[16] T. Yamano, Y. Kaneda, S. Huang, S. H. Hiramatsu, and D. S. B. Hoon, "Enhancement of immunity by a DNA melanoma vaccine against TRP2 with CCL21 as an adjuvant," Molecular Therapy, vol. 13, no. 1, pp. 194-202, 2006.

[17] C.-M. Liang, C.-P. Zhong, R.-X. Sun et al., "Local expression of secondary lymphoid tissue chemokine delivered by adenoassociated virus within the tumor bed stimulates strong antiliver tumor immunity," Journal of Virology, vol. 81, no. 17, pp. 9502-9511, 2007.

[18] C. L. Trimble, R. A. Clark, C. Thoburn et al., "Human papillomavirus 16-associated cervical intraepithelial neoplasia in humans excludes CD8 T cells from dysplastic epithelium," The Journal of Immunology, vol. 185, no. 11, pp. 7107-7114, 2010. 
[19] G. M. P.G. Attianese, V. Marin, V. Hoyos et al., "In vitro and in vivo model of a novel immunotherapy approach for chronic lym phocytic leukemia by anti-CD23 chimeric antigen receptor," Blood, vol. 117, no. 18, pp. 4736-4745, 2011.

[20] Q. Ghia, C. Wang, X. Yuan, Z. Feng, and Z. Han, "Prognostic value of tumor-infiltrating lymphocytes for patients with head and neck squamous cell carcinoma," Translational Oncology, vol. 10, no. 1, pp. 10-16, 2017.

[21] S. E. Stanton and M. L. Disis, "Clinical significance of tumorinfiltrating lymphocytes in breast cancer," Journal for Immunotherapy of Cancer, vol. 4, no. 1, p. 59, 2016.

[22] N. Lee, L. R. Zakka, M. C. Mihm, and T. Schatton, "Tumourinfiltrating lymphocytes in melanoma prognosis and cancer immunotherapy," Pathology, vol. 48, no. 2, pp. 177-187, 2016.

[23] C. Walesky and U. Apte, "Role of hepatocyte nuclear factor $4 \alpha$ (HNF4 $\alpha$ ) in cell proliferation and cancer," Gene Expression, vol. 16, no. 3, pp. 101-108, 2015.

[24] L. Wei, Y. Dai, Y. Zhou et al., "Oroxylin A activates PKM1/ HNF4 alpha to induce hepatoma differentiation and block cancer progression," Cell Death \& Disease, vol. 8, no. 7, Article ID e2944, 2017.

[25] B. Lucas, K. Grigo, S. Erdmann, J. Lausen, L. Klein-Hitpass, and G. U. Ryffel, "HNF $4 \alpha$ reduces proliferation of kidney cells and affects genes deregulated in renal cell carcinoma," Oncogene, vol. 24, no. 42, pp. 6418-6431, 2005.

[26] X.-S. Liu, J. E. Haines, E. K. Mehanna et al., "ZBTB7A acts as a tumor suppressor through the transcriptional repression of glycolysis," Genes \& Development, vol. 28, no. 17, pp. 1917-1928, 2014.

[27] Y. Yang, J. Cui, F. Xue et al., "Pokemon (FBI-1) interacts with Smad4 to repress TGF- $\beta$-induced transcriptional responses," Biochimica et Biophysica Acta (BBA)-Gene Regulatory Mechanisms, vol. 1849, no. 3, pp. 270-281, 2015.

[28] G. Xu, A. Lunardi, J. Zhang et al., "Zbtb7a suppresses prostate cancer through repression of a Sox9-dependent pathway for cellular senescence bypass and tumor invasion," Nature Genetics, vol. 45, no. 7, pp. 739-746, 2013.

[29] G. Sun, B. Peng, Q. Xie, J. Ruan, and X. Liang, "Upregulation of ZBTB7A exhibits a tumor suppressive role in gastric cancer cells," Molecular Medicine Reports, vol. 17, no. 2, pp. 2635-2641, 2018.

[30] X.-S. Liu, M. D. Genet, J. E. Haines et al., “ZBTB7A suppresses melanoma metastasis by transcriptionally repressing MCAM," Molecular Cancer Research, vol. 13, no. 8, pp. 1206-1217, 2015.

[31] X. Wang and Q. Sun, "TP53 mutations, expression and interaction networks in human cancers," Oncotarget, vol. 8, no. 1, pp. 624-643, 2017.

[32] Z. Andrysik, M. D. Galbraith, A. L. Guarnieri et al., "Identification of a core TP53 transcriptional program with highly distributed tumor suppressive activity," Genome Research, vol. 27, no. 10, pp. 1645-1657, 2017.

[33] T. Barnoud, J. L. D. Parris, and M. E. Murphy, “Tumor cells containing the African-Centric S47 variant of TP53 show increased warburg metabolism," Oncotarget, vol. 10, no. 11, pp. 1217-1223, 2019.

[34] W.-N. Zhou, Y.-F. Du, J. Bai et al., "RUNX3 plays a tumor suppressor role by inhibiting cell migration, invasion and angiogenesis in oral squamous cell carcinoma," Oncology Reports, vol. 38, no. 4, pp. 2378-2386, 2017.

[35] X. Ju, T.-O. Ishikawa, K. Naka, K. Ito, Y. Ito, and M. Oshima, "Context-dependent activation of Wnt signaling by tumor suppressor RUNX3 in gastric cancer cells," Cancer Science, vol. 105, no. 4, pp. 418-424, 2014.
[36] L. F. Chen, "Tumor suppressor function of RUNX3 in breast cancer," Journal of Cellular Biochemistry, vol. 113, no. 5, pp. 1470-1477, 2012.

[37] C. Tomasetti and B. Vogelstein, "Variation in cancer risk among tissues can be explained by the number of stem cell divisions," Science, vol. 347, no. 6217, pp. 78-81, 2015.

[38] A. Koch, S. C. Joosten, Z. Feng et al., "Analysis of DNA methylation in cancer: location revisited," Nature Reviews Clinical Oncology, vol. 15, no. 7, pp. 459-466, 2018.

[39] J. X. Binder, S. Pletscher-Frankild, K Tsafou et al., "Compartments: unification and visualization of protein subcellular localization evidence," Database, vol. 2014, 2014.

[40] F. Q. Nuttall, M. C. Gannon, and K. Jordan, "The metabolic response to ingestion of proline with and without glucose," Metabolism, vol. 53, no. 2, pp. 241-246, 2004.

[41] M. Romagnoli, K. Belguise, Z. Yu et al., "Epithelial-to-Mesenchymal transition induced by TGF- $\beta 1$ is mediated by blimp-1-dependent repression of BMP-5," Cancer Research, vol. 72 , no. 23 , pp. $6268-6278,2012$.

[42] T. Sonenshein, C. Lagman, L. K. Chung et al., "Insights into CCL21's roles in immunosurveillance and immunotherapy for gliomas," Journal of Neuroimmunology, vol. 305, pp. 2934, 2017.

[43] F. Tang, J.-N. Chen, N.-N. Zhang et al., "Expression of CCL21 by EBV-associated gastric carcinoma cells protects CD8+ CCR7+ T lymphocytes from apoptosis via the mitochondriamediated pathway," Pathology, vol. 50, no. 6, pp. 613-621, 2018.

[44] K. Liu, C. Zhao, J. Chen et al., "[Overexpression of SEPP1 inhibits the proliferation and induces cell cycle G2/M arrest of 786-O and 769-P human renal carcinoma cells]," Xi bao yu fen $z i$ mian yi xue za zhi = Chinese Journal of Cellular and Molecular Immunology, vol. 32, no. 6, pp. 764-769, 2016.

[45] P. Doolan, M. Clynes, S. Kennedy et al., "TMEM25, REPS2 and Meis 1: favourable prognostic and predictive biomarkers for breast cancer," Tumor Biology, vol. 30, no. 4, pp. 200-209, 2009.

[46] E. Taskesen, S. Babaei, M. M. Reinders, and J. De Ridder, "Integration of gene expression and DNA-methylation profiles improves molecular subtype classification in acute myeloid leukemia," BMC Bioinformatics, vol. 16, no. Suppl 4, p. S5, 2015.

[47] B. Li, B. Qiu, D. S. M. Lee et al., "Fructose-1,6-bisphosphatase opposes renal carcinoma progression," Nature, vol. 513, no. 7517, pp. 251-255, 2014. 